\title{
Erratum
}

Ann H. Maradiegue*, Quannetta T. Edwards, and Diane Seibert

\section{Erratum to 5-Years Later - Have Faculty Integrated Medical Genetics into Nurse Practitioner Curriculum?}

*Corresponding author: Ann H. Maradiegue, Trinity Washington University, 125 Michigan Avenue NE, Washington, DC 20017, USA, E-mail: maradiegura@trinitydc.edu

Quannetta T. Edwards, Western University of Health Sciences, Pomona, CA, USA, E-mail: qedwards@westernu.edu Diane Seibert, Uniformed Services University of the Health Sciences, Bethesda, MD, USA, E-mail: dseibert@usuhs.edu
Erratum in: International Journal of Nursing Education Scholarship 2013;10(1):DOI 10.1515/ijnes-2012-0007. After online publication of ijnes-2012-0007 we realized that some of the data for Tables 2, 3 and 4 were in error. Please find the revised Tables 2, 3, and 4 with correct data.

Table 2 Advance practice (APRN) faculty's self-reported level of comfort teaching genetic/genomic concepts and conditions - years 2010 and $2005(N=85$ and $N=40$, respectively)

Genetic/genomic condition \& concepts

\begin{tabular}{|c|c|c|c|c|}
\hline \multicolumn{2}{|c|}{ Year 2010} & \multicolumn{2}{|c|}{ Year 2005} & \multirow{2}{*}{$\begin{array}{l}\text { **Percent difference } \\
\text { in } 2010 \& 2005^{*}\end{array}$} \\
\hline Yes & No & Yes & No & \\
\hline$N$ (\%) & $N(\%)$ & $N(\%)$ & $N(\%)$ & $\begin{array}{l}\text { Yes to comfort in teaching } \\
\text { genetics/genomics (\%) }\end{array}$ \\
\hline
\end{tabular}

Basic genetic concepts Autosomal dominant Autosomal recessive

X-linked

Mitochondrial inheritance

Three-generation pedigree

Genetic/genomic conditions

Tay-Sachs disease

Cystic fibrosis

Breast/ovarian cancer

Colon cancer

Hemochromatosis

Sickle cell anemia

Thalessemia

Fragile- $X$ syndrome

Huntington's disease

Phenylketonuria

Gaucher's disease

Myotonic dystrophy

Klinefleter's syndrome

Diabetes and genetics

Cardiovascular conditions and genetics

Trisomy 13, 18 or 21

Familial hypercholesterolemia

Other

Polymerase chain reaction

Gene therapy

Online Inheritance in Man (OMIM)
59 (69.4) $60(70.6)$

$26(30.6)$
$25(29.4)$
$29(34.1)$
$43(50.6)$
$18(21.2)$

$35(41.2)$
$26(30.6)$
$1(1.0)$
$14(16.4)$
$14(16.4)$
$10(11.8)$
$15(17.6)$
$39(45.8)$
$30(35.3)$
$36(42.4)$
$57(67.0)$
$54(63.4)$
$39(45.9)$
$39(45.9)$
$36(42.4)$
$37(43.6)$
$30(39.3)$

$23(58.0)$

23 (58.0)

22 (55.0)

$56(65.9)$

42 (49.4)

67 (78.8)

$50(58.8)$

59 (69.4)

84 (99.0)

71 (83.6)

71 (83.6)

75 (88.2)

70 (82.4)

$46(54.2)$

$55(64.7)$

$49(57.6)$

28 (33.0)

31 (36.6)

$46(54.1)$

$46(54.1)$

$49(57.6)$

$48(56.4)$

55 (60.7)

20 (23.5)

33 (38.8)

$30(35.3)$

$16(40.0)$

$24(60.0)$

65 (76.5)

$52(61.2)$

55 (64.7)
$17(42.0)$

17 (42.0)

$18(46.0)$

$30(75.0)$

$24(60.0)$
$15(37.0)$

$18(45.0)$

23 (58.0)

22 (55.0)

16 (35.4)

$22(55.0)$

$20(50.0)$

14 (35.0)

19 (48.0)

$6(15.0)$

$11(28.0)$

14 (35.0)

$22(55.0)$

21 (53.0)

17 (43.0)

19 (48.0)

25 (63.0)

$22(55.0)$

$17(42.0)$

$18(45.0)$

24 (64.6)

$16(40.0)$

$18(45.0)$

$20(50.0)$

$26(65.0)$

$21(52.0)$

34 (85.0)

$29(72.0)$

$26(65.0)$

$18(45.0)$

$19(48.0)$

$23(58.0)$

21 (52.0)

$6(15.0) \quad 34(85.0)$

29 (73.0)

$\mathrm{N} / \mathrm{A}$
11.4

12.6

10.9

24.4

38.8

21.8

24.4

41.0

38.6

48.2

28.2

27.4

4.2

29.7

9.6

18.0

8.6

19.1

$-0.9$

4.6

23.4

12.7

8.5

10.8

Notes: ${ }^{2} 2005$ data from Edwards, Maradiegue, Seibert, Macri, \& Sitzer. (2006). Faculty member’s perceptions of medical genetics and its integration into advance practice curricula. Journal of Nursing Education, 45(3), 128.

**Percent change reflects "high-level" of integration into advance practice curriculum years 2010 compared to 2006; N/A: no data obtained. 
Table 3 Advance practice (APRN) faculty's self-reported level of integration of genetic concepts and conditions into advance practice curricula - years 2010 and $2005(N=85 / N=40$, respectively)

\begin{tabular}{|c|c|c|c|c|c|c|c|}
\hline \multirow[t]{4}{*}{ Genetic/genomic topics } & & & & \multicolumn{3}{|c|}{ Level of integration } & \multirow{4}{*}{$\begin{array}{r}\text { **Percent change } \\
\text { for high-level } \\
\text { integration (\%) }\end{array}$} \\
\hline & \multirow[b]{2}{*}{ None } & \multicolumn{2}{|c|}{ Year - 2010} & \multicolumn{3}{|c|}{ Year - 2005* } & \\
\hline & & Minimal & High & None & Minimal & High & \\
\hline & $N(\%)$ & $N(\%)$ & $N(\%)$ & $N(\%)$ & $N(\%)$ & $N(\%)$ & \\
\hline \multicolumn{8}{|l|}{ Basic genetic concepts } \\
\hline Autosomal dominant & $5(6.4)$ & $57(66.5)$ & $23(27.1)$ & $6(15.0)$ & $25(62.0)$ & $9(23.0)$ & 4.1 \\
\hline Autosomal recessive & $6(7.1)$ & $56(65.8)$ & $23(27.1)$ & $6(15.0)$ & $25(62.0)$ & $9(23.0)$ & 4.1 \\
\hline X-linked & $8(9.4)$ & $60(70.7)$ & $17(19.9)$ & $7(17.0)$ & $25(63.0)$ & $8(20.0)$ & -0.1 \\
\hline Mitochondrial inheritance & $12(14.1)$ & $61(71.8)$ & $12(14.1)$ & $17(42.0)$ & $20(50.0)$ & $3(8.0)$ & 6.1 \\
\hline Three-generation pedigree & $5(6.9)$ & $43(54.8)$ & $37(38.3)$ & $8(20.0)$ & $22(55.0)$ & $10(25.0)$ & $13.3^{* \star}$ \\
\hline \multicolumn{8}{|l|}{ Genetic/genomic conditions } \\
\hline Tay-Sachs disease & $5(6.4)$ & $63(73.6)$ & $17(20.0)$ & $12(30.0)$ & $23(57.0)$ & $5(13.0)$ & 7.0 \\
\hline Cystic fibrosis & $2(2.0)$ & $58(65.0)$ & $25(33.0)$ & $6(15.0)$ & $21(54.7)$ & $13(30.3)$ & 2.7 \\
\hline Breast/ovarian cancer & $1(1.2)$ & $48(54.8)$ & $36(44.0)$ & $3(7.0)$ & $21(53.0)$ & $16(40.0)$ & 4.0 \\
\hline Colon cancer & $2(2.4)$ & $48(56.4)$ & $35(41.2)$ & $6(15.0)$ & $20(50.0)$ & $14(35.0)$ & 6.2 \\
\hline Hemochromatosis & $11(12.9)$ & $28(32.9)$ & $46(54.2)$ & $17(42.0)$ & $20(50.0)$ & $3(8.0)$ & $46.2^{\star \star}$ \\
\hline Sickle cell anemia & $2(2.0)$ & $46(49.8)$ & $37(48.2)$ & $5(13.0)$ & $23(57.0)$ & $12(30.0)$ & $18.2^{\star \star}$ \\
\hline Thalessemia & $3(3.5)$ & $61(71.8)$ & $21(24.7)$ & $9(23.0)$ & $22(54.5)$ & $9(22.5)$ & 2.2 \\
\hline Fragile- $X$ syndrome & $11(12.9)$ & $59(69.9)$ & $15(17.2)$ & $14(35.0)$ & $20(50.0)$ & $6(15.0)$ & 2.2 \\
\hline Huntington's disease & $6(7.1)$ & $65(76.5)$ & $14(16.4)$ & $14(35.0)$ & $23(57.0)$ & $3(8.0)$ & 8.4 \\
\hline Phenylketonuria & $5(6.0)$ & $44(55.2)$ & $36(38.8)$ & $14(35.0)$ & $15(37.0)$ & $11(28.0)$ & 10.8 \\
\hline Gaucher's disease & $27(31.8)$ & $52(61.1)$ & $6(7.1)$ & $30(75.0)$ & $1(2.0)$ & $9(23.0)$ & -15.9 \\
\hline Myotonic dystrophy & $25(29.4)$ & $52(61.1)$ & $8(9.5)$ & $24(60.0)$ & $14(35.0)$ & $2(5.0)$ & 4.5 \\
\hline Klinefleter's syndrome & $10(11.8)$ & $57(67.0)$ & $18(21.2)$ & $16(40.0)$ & $19(47.0)$ & $5(13.0)$ & 8.2 \\
\hline Diabetes and genetics & $11(12.9)$ & $58(61.9)$ & $16(25.2)$ & $3(8.0)$ & $22(54.0)$ & $15(38.0)$ & -12.8 \\
\hline Cardiovascular conditions and genetics & $7(8.2)$ & $29(34.1)$ & $49(57.7)$ & $5(13.0)$ & $21(52.0)$ & $14(35.0)$ & $22.7^{\star \star}$ \\
\hline Trisomy 13,18 or 21 & $10(11.8)$ & $57(64.6)$ & $18(23.6)$ & $13(33.0)$ & $19(47.0)$ & $8(20.0)$ & 3.6 \\
\hline Familial hypercholesterolemia & $7(8.2)$ & $48(56.5)$ & $30(35.3)$ & $13(33.0)$ & $14(34.0)$ & $13(33.0)$ & 2.3 \\
\hline \multicolumn{8}{|l|}{ Other } \\
\hline Polymerase chain reaction & $28(32.9)$ & $45(52.9)$ & $12(14.2)$ & $29(72.0)$ & $7(18.0)$ & $4(10.0)$ & 4.2 \\
\hline Gene therapy & $13(15.3)$ & $65(74.4)$ & 7 (10.3) & $20(50.0)$ & $16(40.0)$ & $4(10.0)$ & 0.3 \\
\hline
\end{tabular}

Notes: *2005 data from Edwards, Maradiegue, Seibert, Macri, \& Sitzer. (2006). Journal of Nursing Education, 45(3), 128. Faculty member’s perceptions of medical genetics and its integration into advance practice curricula.

**Percent change reflects "high-level" of integration into advance practice curriculum years 2010 compared to 2006. 
Table 4 Advance practice (APRN) faculty who self-reported formal education or training in genetics/genomics in years 2010 and 2005 $(N=85$ and $N=40$, respectively)

\begin{tabular}{|c|c|c|c|c|c|}
\hline \multirow[t]{3}{*}{ Genetic/genomic concepts $\&$ conditions } & \multicolumn{2}{|r|}{ Year 2010} & \multicolumn{2}{|r|}{ *Year 2005} & \multirow{3}{*}{$\begin{array}{r}\% \text { Difference } \\
\text { "Yes" faculty training/educatior } \\
\text { Year } 2010 \text { and } 200\end{array}$} \\
\hline & Yes & No & Yes & No & \\
\hline & $N(\%)$ & $N(\%)$ & $N(\%)$ & $N(\%)$ & \\
\hline \multicolumn{6}{|l|}{ Basic genetic concepts } \\
\hline Autosomal dominant & $6(7.1)$ & 79 (92.9) & $15(37.5)$ & $25(62.5)$ & $-30.4 \%$ \\
\hline Autosomal recessive & $3(3.5)$ & $82(96.5)$ & $15(37.5)$ & $25(62.5)$ & $-34.0 \%$ \\
\hline X-linked & $3(3.5)$ & $82(96.5)$ & $15(37.0)$ & $25(63.0)$ & $-33.5 \%$ \\
\hline Mitochondrial inheritance & $7(8.2)$ & $78(91.8)$ & $8(20.0)$ & $32(80.0)$ & $-11.8 \%$ \\
\hline Three-generation pedigree & $7(8.2)$ & $78(91.8)$ & $13(32.5)$ & $27(67.5)$ & $-24.3 \%$ \\
\hline \multicolumn{6}{|l|}{ Genetic/Genomic conditions } \\
\hline Tay-Sachs disease & $4(4.7)$ & $81(95.3)$ & $9(22.5)$ & $31(77.5)$ & -17.8 \\
\hline Cystic fibrosis & $4(4.7)$ & $81(95.3)$ & $10(25.0)$ & $30(75.0)$ & -20.3 \\
\hline Breast/ovarian cancer & $4(4.7)$ & $81(95.3)$ & $\star \star 7(18.5)$ & $\star \star 31(81.5)$ & -13.8 \\
\hline Colon cancer & $4(4.7)$ & $81(95.3)$ & $9(22.5)$ & $31(77.5)$ & -17.8 \\
\hline Hemochromatosis & $5(5.9)$ & $80(94.1)$ & $6(15.0)$ & $34(85.0)$ & -9.1 \\
\hline Sickle cell anemia & $4(4.7)$ & $81(95.3)$ & $9(22.5)$ & $31(77.5)$ & -17.8 \\
\hline Thalessemia & $3(3.5)$ & $82(96.5)$ & $11(27.5)$ & $29(72.5)$ & -24.0 \\
\hline Fragile- $X$ syndrome & $4(4.7)$ & $81(95.3)$ & $10(25.0)$ & $30(75.0)$ & -20.3 \\
\hline Huntington's disease & $4(4.7)$ & $81(95.3)$ & $8(20.0)$ & $32(80.0)$ & -15.3 \\
\hline Phenylketonuria & $4(4.7)$ & $81(95.3)$ & $8(20.0)$ & $32(80.0)$ & -15.3 \\
\hline Gaucher's disease & $3(3.5)$ & $82(96.5)$ & $4(10.0)$ & $36(90.0)$ & -6.5 \\
\hline Myotonic dystrophy & $3(3.5)$ & $82(96.5)$ & $7(17.5)$ & $33(82.5)$ & -14.0 \\
\hline Klinefleter's syndrome & $4(4.7)$ & $81(95.3)$ & $9(22.5)$ & $31(77.5)$ & -17.8 \\
\hline Diabetes and genetics & $4(4.7)$ & $81(95.3)$ & $9(22.5)$ & $31(77.5)$ & -17.8 \\
\hline Cardiovascular conditions and genetics & $4(4.7)$ & $82(96.5)$ & $9(22.5)$ & $31(77.5)$ & -17.8 \\
\hline Trisomy 13,18 or 21 & $2(2.4)$ & $83(97.6)$ & $11(27.5)$ & $29(72.5)$ & -25.1 \\
\hline Familial hypercholesterolemia & $3(3.5)$ & $82(96.5)$ & $8(20.0)$ & $32(80.0)$ & -16.5 \\
\hline \multicolumn{6}{|l|}{ Other } \\
\hline Polymerase chain reaction & $4(4.7)$ & $81(95.8)$ & $5(12.5)$ & $35(87.5)$ & -7.8 \\
\hline Gene therapy & $4(4.7)$ & $81(95.8)$ & $7(17.5)$ & $33(82.5)$ & -12.8 \\
\hline
\end{tabular}

Notes: *2005 data from Edwards, Maradiegue, Seibert, Macri, \& Sitzer. (2006). Faculty member’s perceptions of medical genetics and its integration into advance practice curricula. Journal of Nursing Education, 45(3), 128.

**Denotes missing data. 
